\title{
Papel do cigarro eletrónico na cessação tabágica: uma revisão baseada na evidência
}

Pedro Couto, ${ }^{1 *}$ Pedro Mendes, ${ }^{1 *}$ Rosa Barreira, ${ }^{1 *}$ Joana de Oliveira e Silva, ${ }^{1 *}$ Lígia Silva, ${ }^{1 *}$ Elisabete Almeida ${ }^{2 *}$

\section{RESUMO}

Objetivos: Determinar se a utilização de cigarros eletrónicos (CE) constitui uma alternativa no apoio à cessação tabágica (CT). Fontes de dados: Bases de dados MEDLINE; National Clearinghouse; Canadian Medical Association Practice Guidelines InfoBase; Guidelines Finder da National Electronic Library for Health do NHS britânico; Database of Abstracts of Reviews of Effectiveness - Centre for Reviews and Dissemination; Bandolier; The Cochrane Library; Índex de Revistas Médicas Portuguesas.

Métodos de revisão: Pesquisa de artigos publicados entre 01/07/2004 e 30/06/2014, utilizando o termo MeSH smoking cessation, os termos electronic cigarette(s), e-cigarette(s), e-cig(s) e em português "cessação tabágica" e "cigarro(s) ele(c)trónico(s)". Foram incluídos estudos que avaliassem a CT em fumadores expostos e não expostos ao CE. As escalas Strength-of-Recommendation Taxonomy, Jadad e Newcastle-Ottawa foram utilizadas para avaliar a qualidade dos estudos e a força de recomendação.

Resultados: Da pesquisa resultaram 2.076 artigos; 10 foram excluídos por serem repetidos, 2.005 após leitura do título por não estarem relacionados diretamente com o tema, 47 após leitura do resumo e 11 após leitura integral por não satisfazerem os critérios de inclusão. Foram assim incluídos três artigos: uma revisão sistemática que incluiu nove estudos originais [Nível de Evidência (NE) 2]; uma revisão clássica (NE 3), cujas conclusões foram desfavoráveis à utilização dos CE; e um estudo transversal que mostrou uma maior utilização de CE em relação a outras terapêuticas entre aqueles que atingiram CT (NE 3).

Conclusões: A maioria dos trabalhos não encontrou associação entre o uso de CE e a CT. Os autores consideram que o seu uso não parece desempenhar um papel benéfico na CT (Força de Recomendação B). Dada a rápida expansão e crescente popularidade destes produtos, são necessários estudos de melhor qualidade.

Palavras-chave: Cigarro Eletrónico; Cessação Tabágica.

\section{INTRODUÇÃO}

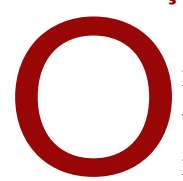

$\mathrm{s}$ cigarros eletrónicos foram criados na China, em 2004, pela empresa Ruyan, ${ }^{1}$ consistindo em dispositivos elétricos que libertam nicotina através de uma mistura de vapor. Com a inalação é ativado um atomizador, responsável pelo aquecimento de uma solução de nicotina (geralmente com propilenoglicol ou glicerina), produzindo um vapor que depois é inalado. Adicionalmente à nicotina, o vapor pode conter sabores ou aromas e provocar uma sensação semelhante ao ato de fumar sem que, no entanto, haja fumo ou combustão envolvidos. ${ }^{2-3}$

Embora o cigarro eletrónico tenha sido desenvolvido

'Médico Interno de Medicina Geral e Familiar, USF Valongo, ACES Grande Porto III - Maia/Valongo

Médica Assistente de Medicina Geral e Familiar, USF Valongo, ACES Grande Porto III - Maia/Valongo

* Os autores contribuíram de igual forma para a elaboração do presente trabalho. e inicialmente publicitado de forma independente da indústria tabaqueira, as quatro maiores empresas multinacionais deste ramo já possuem ou estão a desenvolver um produto deste tipo. ${ }^{4}$ A primeira a entrar neste ramo foi a Lorillard Inc. que adquiriu a marca Blu-Cigs em abril de 2012. ${ }^{5}$ Estima-se que as receitas provenientes da venda de cigarros eletrónicos atinjam cerca de 1,5 biliões de dólares no ano de 2014 . $^{6}$

O conhecimento da população sobre este tipo de dispositivos e a sua utilização variam consideravelmente entre países; contudo, há uma tendência clara para o aumento da sua popularidade ao longo do tempo., ${ }^{1,7} \mathrm{Em} 2012$, cerca de $70 \%$ dos cidadãos europeus já tinha ouvido falar de cigarros eletrónicos e 7\% já os tinham utilizado pelo menos uma vez, ${ }^{1,8}$ valores que não diferem muito daqueles relativos aos Estados Unidos da América., ${ }^{5,9}$ Já em Portugal, dados de 2012 apontam para valores inferiores à média europeia: $58 \%$ dos portugueses já ouviu falar de cigarros eletrónicos 
e $4 \%$ já os experimentaram. ${ }^{8} \mathrm{O}$ aumento da popularidade destes dispositivos nas pesquisas online foi também demonstrado. ${ }^{10} \mathrm{~A}$ Internet parece ser o principal canal de disseminação de informação e venda destes produtos. ${ }^{11}$

Ao contrário dos produtos derivados do tabaco, os cigarros eletrónicos não têm restrições legais que impeçam a sua publicitação. As empresas que os comercializam anunciam-nos como uma opção mais económica, mais segura e saudável, fazendo frequentemente referência não só ao seu papel na cessação tabágica, mas também à possibilidade de se fumar em locais proibidos, sem as implicações da exposição passiva ao fumo do cigarro convencional. ${ }^{11-12}$ Da mesma forma, as razões que levam as pessoas a utilizar estes produtos já foram estudadas, estando em consonância com as alegadas vantagens e benefícios anunciados pelas empresas. A maioria utiliza-os para deixar de fumar e por considerar que são menos tóxicos do que os cigarros convencionais..$^{2-3,3,13-15} \mathrm{~A}$ possibilidade de fumar em zonas proibidas e o preço mais baixo são outros pontos atrativos. ${ }^{2,13}$

A regulamentação deste produto tem sido sistematicamente adiada e alvo de polémica devido à ambiguidade da sua classificação em dispositivo médico ou produto derivado do tabaco. ${ }^{15}$ No entanto, em fevereiro de 2014, o Parlamento Europeu aprovou uma nova diretiva, definindo apenas os cigarros eletrónicos que contenham dosagens de nicotina até $20 \mathrm{mg} / \mathrm{ml}$ como produtos derivados do tabaco, estabelecendo algumas regras de segurança e de embalagem. Assim, os dispositivos com dosagens superiores, ou que aleguem benefício para a saúde, terão que ser licenciados como medicamentos. A aplicação destas novas regras está prevista para 2016, não havendo no entanto imposições relativas à idade mínima legal ou à sua utilização em espaços fechados. ${ }^{16}$

Assim, dada a utilização destes dispositivos na cessação tabágica, os autores deste trabalho têm como objetivo determinar se a utilização de cigarros eletrónicos, à luz da evidência atual, constitui uma alternativa no apoio à cessação tabágica.

\section{MÉTODOS}

Foi realizada uma pesquisa de artigos (meta-análises, revisões sistemáticas, estudos originais, normas de orientação clínica), publicados entre 1 de julho de 2004 e 30 de junho de 2014 nas bases de dados MEDLINE; National Clearinghouse; Canadian Medical Association Practice Guidelines InfoBase; Guidelines Finder da National Electronic Library for Health do NHS britânico; Databa- se of Abstracts of Reviews of Effectiveness - Centre for Reviews and Dissemination; Bandolier; The Cochrane Library; e Índex de Revistas Médicas Portuguesas.

Para efetuar a pesquisa foi usado o termo MeSH Smoking Cessation. Perante a inexistência de um termo MeSH referente a cigarros eletrónicos, foram também utilizados os seguintes termos: electronic cigarette, e-cigarette, e-cig, electronic cigarettes, e-cigarettes e e-cigs. Foram ainda usados os termos portugueses "cessação tabágica", "cigarros eletrónicos" e "cigarros electrónicos". A pesquisa foi limitada a artigos na língua portuguesa, inglesa e espanhola.

Foram incluídos artigos cujo estudo incidisse numa população de fumadores, com um grupo exposto ao cigarro eletrónico (intervenção) comparativamente a um grupo que não utiliza cigarro eletrónico [terapêutica de substituição de nicotina (TSN), vareniclina/bupropiona, placebo/ausência de tratamento]. O resultado medido foi a cessação tabágica definida pela não utilização de produtos derivados do tabaco.

Utilizou-se a escala Strength of Recommendation Taxonomy (SORT), da American Academy of Family Physicians, na avaliação do nível de evidência e atribuição de força de recomendação. ${ }^{17}$ Para avaliação da qualidade dos trabalhos originais incluídos foi utilizada a escala de Jadad para os ensaios clínicos aleatorizados e controla$\operatorname{dos}^{18} \mathrm{e}$ as várias versões da escala Newcastle-Ottawa para os estudos observacionais. ${ }^{19}$

A seleção dos trabalhos pelo título e resumo foi dividida entre todos os autores. Os artigos selecionados para leitura integral foram lidos por, pelo menos, três dos autores para decidir a sua inclusão. A avaliação final da qualidade e nível de evidência dos artigos incluídos foi discutida e decidida em reunião com todos os autores.

\section{RESULTADOS}

Da pesquisa realizada resultou um total de 2.076 artigos, tendo sido apenas incluídos três: uma revisão sistemática com meta-análise, uma revisão clássica e um estudo transversal. O fluxograma de seleção dos estudos está representado na figura 1. A maioria dos artigos foi excluída após leitura do título por não estarem relacionados diretamente com o tema, tendo contribuído para esta situação o facto de uma das fontes de pesquisa ter produzido resultados que não incluíam os termos pesquisados. A qualidade dos artigos presentes na revisão sistemática com meta-análise foi avaliada para a determinação do nível de evidência da revisão, não tendo sido considerados individualmente 


\section{QUADRO I. Resumo da avaliação da qualidade dos estudos incluídos na revisão sistemática com metanálise}

\begin{tabular}{|c|c|c|c|c|c|c|}
\hline $\begin{array}{l}\text { Autor } \\
\text { (Ano) }\end{array}$ & Tipo de estudo & População/Métodos & $\begin{array}{l}\text { Definição de cessação } \\
\text { tabágica }\end{array}$ & Conclusão & $\begin{array}{c}\text { NCO } \\
\text { (máx. 9) }\end{array}$ & $\begin{array}{l}\text { JADAD } \\
\text { (máx. 5) }\end{array}$ \\
\hline $\begin{array}{l}\text { Adkison SE, } \\
\text { et al (2013) }\end{array}$ & $\begin{array}{l}\text { Observacional } \\
\text { Longitudinal }\end{array}$ & $\begin{array}{l}\text { Questionário telefónico } \\
\text { a fumadores e } \\
\text { ex-fumadores. Primeiro } \\
\text { questionário aplicado } \\
\text { em } 2008 / 2009 \text {, } \\
\text { segundo em } 2010 / 2011 \\
N=4.136\end{array}$ & $\begin{array}{l}\text { Auto-reportada, } \\
\text { definida como não } \\
\text { utilização de tabaco no } \\
\text { momento do } \\
\text { questionário }\end{array}$ & $\begin{array}{l}\text { Sem diferença na } \\
\text { cessação entre os } \\
\text { grupos com e sem } \\
\text { utilização de CE } \\
\text { OR: } 0,81[95 \% \\
\text { IC: } 0,43-1,53] p=0,52\end{array}$ & 4 & NA \\
\hline $\begin{array}{l}\text { Grana R, et } \\
\text { al (2014) }\end{array}$ & $\begin{array}{l}\text { Observacional } \\
\text { Longitudinal }\end{array}$ & $\begin{array}{l}\text { Questionário a } \\
\text { fumadores no início e } \\
\text { após um ano de } \\
\text { seguimento } \\
\mathrm{N}=949\end{array}$ & $\begin{array}{l}\text { Auto-reportada no } \\
\text { segundo questionário, } \\
\text { não descrita definição } \\
\text { de cessação tabágica }\end{array}$ & $\begin{array}{l}\text { Uso de CE no início do } \\
\text { estudo não permitiu } \\
\text { predizer a taxa de } \\
\text { cessação um ano } \\
\text { depois } \\
\text { OR: } 0,76 \text { [95\% IC: } \\
0,36-1,60]\end{array}$ & 6 & NA \\
\hline $\begin{array}{l}\text { Vickerman } \\
\text { KA, et al } \\
(2013)\end{array}$ & $\begin{array}{l}\text { Observacional } \\
\text { Longitudinal }\end{array}$ & $\begin{array}{l}\text { Questionário a } \\
\text { fumadores sete meses } \\
\text { após terem utilizado } \\
\text { uma linha telefónica de } \\
\text { apoio à cessação } \\
\text { tabágica } \\
\mathrm{N}=2.758 \\
\text { Idade média: } 48,6 \text { anos }\end{array}$ & $\begin{array}{l}\text { Auto-reportada, } \\
\text { definida como não } \\
\text { utilização de tabaco } \\
\text { nos } 30 \text { dias anteriores } \\
\text { ao questionário }\end{array}$ & $\begin{array}{l}\text { Aos } 7 \text { meses, os } \\
\text { utilizadores de CE } \\
\text { apresentaram uma } \\
\text { menor probabilidade } \\
\text { de abstinência } \\
\text { OR: } 0,50 \text { [95\% IC: } \\
0,40-0,63]\end{array}$ & 3 & NA \\
\hline $\begin{array}{l}\text { Choi K, et } \\
\text { al (2014) - } \\
\text { resposta }\end{array}$ & $\begin{array}{l}\text { Observacional } \\
\text { Longitudinal }\end{array}$ & $\begin{array}{l}\text { Questionário a } \\
\text { fumadores no início e } \\
\text { após um ano de } \\
\text { seguimento } \\
\mathrm{N}=346 \\
\text { Idade média: } 24,1 \text { anos }\end{array}$ & $\begin{array}{l}\text { Auto-reportada, } \\
\text { definida como não } \\
\text { utilização de tabaco } \\
\text { nos } 30 \text { dias anteriores } \\
\text { ao questionário }\end{array}$ & $\begin{array}{l}\text { Sem diferença na } \\
\text { cessação entre } \\
\text { utilizadores e não } \\
\text { utilizadores de CE } \\
\text { OR: } 0,93 \text { [95\% IC: } \\
0,19-4,63] p=0,93\end{array}$ & 4 & NA \\
\hline $\begin{array}{l}\text { Popova L, } \\
\text { et al (2013) }\end{array}$ & $\begin{array}{l}\text { Observacional } \\
\text { Transversal }\end{array}$ & $\begin{array}{l}\text { Questionário a } \\
\text { fumadores e } \\
\text { ex-fumadores } \\
\mathrm{N}=1.836 \\
\text { Idade média: } 42 \text { anos }\end{array}$ & $\begin{array}{l}\text { Auto-reportada, } \\
\text { definida como não } \\
\text { utilização de tabaco no } \\
\text { momento do } \\
\text { questionário (incluídos } \\
\text { apenas ex-fumadores } \\
\text { há menos de dois anos) }\end{array}$ & $\begin{array}{l}\text { Utilizadores de CE } \\
\text { apresentaram uma } \\
\text { menor probabilidade } \\
\text { de serem ex-fumadores } \\
\text { relativamente aos não } \\
\text { utilizadores } \\
\text { OR: } 0,69 \text { [95\% IC: } \\
0,52-0,94]\end{array}$ & 5 & NA \\
\hline $\begin{array}{l}\text { Polosa R, et } \\
\text { al (2011) }\end{array}$ & $\begin{array}{l}\text { EC não } \\
\text { controlado }\end{array}$ & $\begin{array}{l}\text { Fumadores sem } \\
\text { intenção de deixar de } \\
\text { fumar dentro de } 30 \text { dias } \\
\text { Intervenção: } \mathrm{CE} \\
\text { Follow-up: } 6 \text { meses } \\
\mathrm{N}=40 \\
\text { Idade média: } 42,9 \text { anos }\end{array}$ & $\begin{array}{l}\text { Auto-reportada (não } \\
\text { utilização de tabaco } \\
\text { nos últimos } 30 \text { dias) e } \\
\text { medição de monóxido } \\
\text { de carbono exalado } \\
\text { ( } \leq 10 \text { ppm) }\end{array}$ & $\begin{array}{l}\text { Participantes aos } 6 \\
\text { meses: } 68 \%(N=27) \\
33 \%(N=9) \text { pararam } \\
\text { de fumar tabaco, mas } \\
\text { mantiveram CE }\end{array}$ & NA & NA \\
\hline
\end{tabular}




\section{QUADRO I. (continuação)}

\begin{tabular}{|c|c|c|c|c|c|c|}
\hline $\begin{array}{l}\text { Autor } \\
\text { (Ano) }\end{array}$ & Tipo de estudo & População/Métodos & $\begin{array}{l}\text { Definição de cessação } \\
\text { tabágica }\end{array}$ & Conclusão & $\begin{array}{c}\text { NCO } \\
\text { (máx. 9) }\end{array}$ & $\begin{array}{l}\text { JADAD } \\
\text { (máx. 5) }\end{array}$ \\
\hline $\begin{array}{l}\text { Caponnetto } \\
\text { P, et al } \\
(2013)\end{array}$ & $\begin{array}{l}\text { EC não } \\
\text { controlado }\end{array}$ & $\begin{array}{l}\text { Fumadores com } \\
\text { esquizofrenia sem } \\
\text { intenção de deixar de } \\
\text { fumar dentro de } 30 \text { dias } \\
\text { Intervenção: } \mathrm{CE} \\
\text { Follow-up: } 1 \text { ano } \\
\mathrm{N}=14 \\
\text { Idade média: } 44,6 \text { anos }\end{array}$ & $\begin{array}{l}\text { Auto-reportada (não } \\
\text { utilização de tabaco nos } \\
\text { últimos } 30 \text { dias) e } \\
\text { medição de monóxido } \\
\text { de carbono exalado } \\
\text { ( } \leq 10 \text { ppm) }\end{array}$ & $\begin{array}{l}14,3 \%(N=2) \text { em } \\
\text { abstinência no final do } \\
\text { estudo }\end{array}$ & NA & NA \\
\hline $\begin{array}{l}\text { Caponnetto } \\
\text { P, et al } \\
(2013)\end{array}$ & $\begin{array}{l}\text { EC aleatorizado } \\
\text { controlado }\end{array}$ & $\begin{array}{l}\text { Fumadores sem } \\
\text { intenção de deixar de } \\
\text { fumar } \\
\text { Intervenções: } \\
\text { - CE com 7,2mg de } \\
\text { nicotina } \\
\text { - CE com 5,4mg de } \\
\text { nicotina } \\
\text { - CE sem nicotina } \\
\text { (placebo) } \\
\text { Follow-up: } 1 \text { ano } \\
\text { N = } 300 \\
\text { Idade média: } 44 \text { anos }\end{array}$ & $\begin{array}{l}\text { Auto-reportada (não } \\
\text { utilização de tabaco } \\
\text { nas últimas } 28 \\
\text { semanas) e medição de } \\
\text { monóxido de carbono } \\
\text { exalado ( } \leq 7 \mathrm{ppm} \text { ) }\end{array}$ & $\begin{array}{l}\text { Participantes no final: } \\
61 \%(\mathrm{~N}=183 \text { ) } \\
\text { Sem diferença } \\
\text { estatisticamente } \\
\text { significativa na } \\
\text { cessação entre os } \\
\text { grupos ( } 4 \% \text { para o } \\
\text { placebo; } 9 \% \text { CE de } \\
\text { baixa dose de nicotina } \\
\text { e } 13 \% \text { nos com alta } \\
\text { dose) }\end{array}$ & NA & 5 \\
\hline $\begin{array}{l}\text { Bullen C, } \\
\text { et al (2013) }\end{array}$ & $\begin{array}{l}\text { EC aleatorizado } \\
\text { controlado }\end{array}$ & $\begin{array}{l}\text { Fumadores motivados } \\
\text { a deixar de fumar } \\
\text { Intervenções: } \\
\text { - CE com } 16 \mathrm{mg} \text { de } \\
\text { nicotina } \\
\text { - Sistema transdérmico } \\
\text { com } 21 \mathrm{mg} \text { de nicotina } \\
\text { - CE sem nicotina } \\
\text { Follow-up: } 6 \text { meses } \\
\mathrm{N}=657 \\
\text { Idade media: } 42,1 \text { anos }\end{array}$ & $\begin{array}{l}\text { Auto-reportada (não } \\
\text { utilização de tabaco } \\
\text { durante todo o período } \\
\text { de estudo, permitindo } \\
\leq 5 \text { cigarros no total) e } \\
\text { medição de monóxido } \\
\text { de carbono exalado } \\
\text { ( } \leq 10 \text { ppm) }\end{array}$ & $\begin{array}{l}\text { Participantes aos } 6 \\
\text { meses: } 78 \% \text { ( } \mathrm{N}=513 \text { ) } \\
\text { Análise com intenção } \\
\text { de tratar: sem diferença } \\
\text { estatisticamente } \\
\text { significativa na } \\
\text { cessação entre os } \\
\text { grupos ( } 7,3 \% \text { para CE } \\
\text { com nicotina, 5,8\% } \\
\text { com sistema } \\
\text { transdérmico, } 4,1 \% \\
\text { com CE sem nicotina) }\end{array}$ & NA & 5 \\
\hline
\end{tabular}

Legenda: CE - Cigarros eletrónicos; EC - Ensaio clínico; IC - Intervalo de confiança; N - Número de participantes; NCO - NewCastle-Otawa; NA - Não aplicável; OR - Odds ratio; $p$ - nível de significância estatística.

para a atribuição da força de recomendação final. Essa avaliação pode ser consultada no quadro I.

Grana, et al $^{11}$ incluíram na revisão sistemática um total de nove estudos referentes à utilização de cigarros eletrónicos na cessação tabágica: cinco estudos observacionais e quatro ensaios clínicos. A partir dos estudos observacionais foi então realizada uma meta-análise, concluindo-se que a utilização de cigarros eletrónicos está associada a menor probabilidade de deixar de fumar [odds ratio $(\mathrm{OR})=0,61$; intervalo de confiança (IC) $95 \%$ $=0,50-0,75]$. Da mesma forma, pela análise dos ensaios clínicos concluíram que estes dispositivos não estão associados a uma maior taxa de cessação tabágica.

Odum, et $\mathrm{al}^{20}$ realizaram uma revisão clássica sobre o 


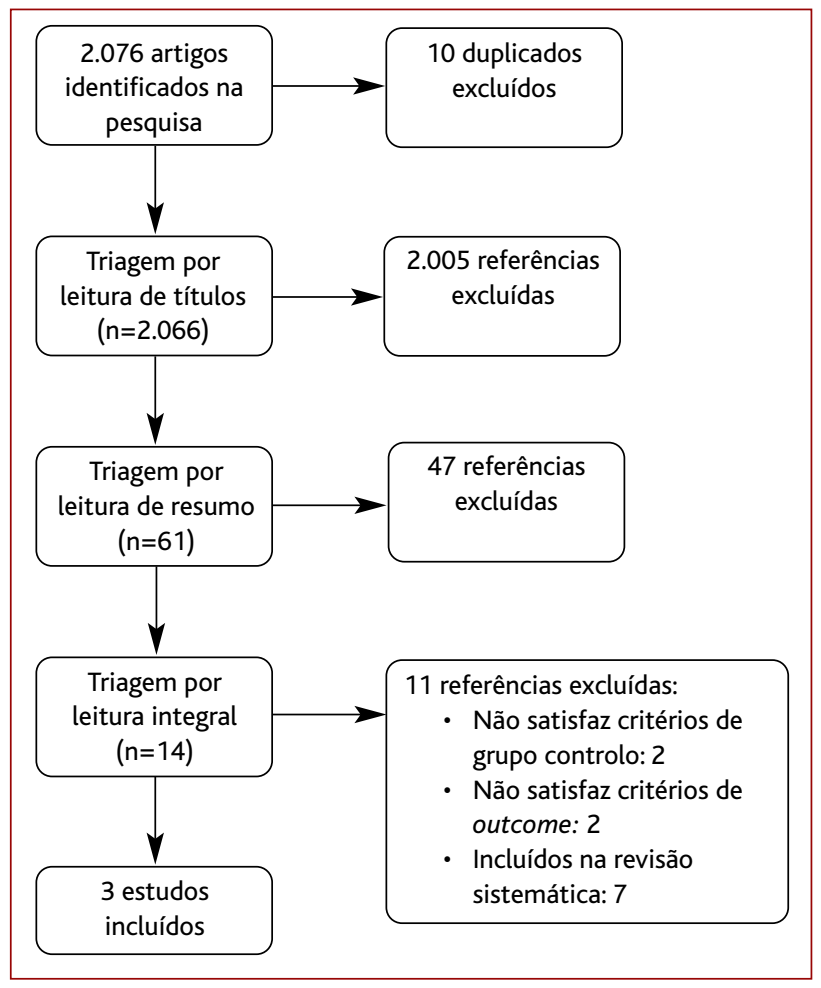

Figura 1. Fluxograma de seleção dos estudos.

tema, concluindo que, apesar de a literatura existente ser promissora, a evidência é escassa sobretudo ao nível de estudos comparativos com as terapêuticas existentes pelo que, até que existam mais estudos e de melhor qualidade, este método não deverá ser recomendado.

O estudo transversal de Brown, et $\mathrm{al}^{21}$ consistiu num inquérito a fumadores (idade média 38,8 anos) que tentaram deixar de fumar nos últimos 12 meses $(n=5.863)$ e mostrou que, entre aqueles que reportaram estar em cessação tabágica à data do inquérito, havia uma maior proporção de utilização de cigarros eletrónicos em relação à utilização de TSN (OR = 1,63; 95\% IC 1,17-2,27) ou à não utilização de qualquer terapêutica $(\mathrm{OR}=1,61 ; \mathrm{IC} 95 \%=1,19-2,18)$.

As principais conclusões e o nível de evidência dos trabalhos incluídos estão sumariados no quadro II.

\section{CONCLUSÕES}

Apesar de alguns estudos terem verificado que os utilizadores consideram que os cigarros eletrónicos os ajudaram a deixar de fumar, ${ }^{13-14}$ a maioria dos trabalhos incluídos não encontrou associação entre o seu uso e o sucesso em atingir a cessação tabágica, apresentando tam- bém limitações importantes.

O estudo com maior nível de evidência, a revisão sistemática com meta-análise de Grana, et al, ${ }_{11}^{11}$ mostrou ter várias limitações, nomeadamente por incluir alguns estudos que não eram de boa qualidade, com populações distintas e achados inconsistentes. Adicionalmente, em todos os estudos observacionais incluídos nessa revisão a cessação tabágica foi considerada atingida quando reportada pelos participantes, não existindo método validado de comprovação deste resultado. Também três dos estudos observacionais incluídos ${ }^{22-24}$ não ajustaram os resultados para o nível de dependência de nicotina e como tal não consideraram que os fumadores mais dependentes podem ser aqueles com maior dificuldade em deixar de fumar. Quanto às limitações dos ensaios clínicos incluídos nesta revisão, dois não tinham grupo de controlo ${ }^{25-26} \mathrm{e}$ naquele com melhor desenho ${ }^{27}$ verificaram-se taxas de abstinência inferiores às previstas, não apresentando poder estatístico suficiente para mostrar diferenças entre os grupos.

No estudo transversal de Popova, et $\mathrm{al}^{24}$ as principais limitações relacionam-se com a cessação tabágica autodeclarada, com o viés de memória e com a heterogeneidade das exposições dos dois grupos analisados.

De referir que alguns autores, ${ }^{28-29}$ tendo em conta a pouca evidência existente, emitem uma opinião cautelosa quanto ao uso do cigarro eletrónico na cessação tabágica, privilegiando as terapêuticas já conhecidas e devidamente aprovadas para essa finalidade. $\mathrm{O}$ Infarmed ${ }^{30}$ publicou ainda uma circular informativa desaconselhando a utilização deste tipo de produtos, por não ser possível assegurar a sua qualidade, segurança, eficácia ou desempenho.

Adicionalmente, devido à rápida expansão do fenómeno dos cigarros eletrónicos em todo o mundo, que já conta com mais de 150 lojas dedicadas em Portugal, ${ }^{31}$ tornase pertinente uma análise cuidada de todas as perspetivas e fatores envolvidos. Algumas vantagens da utilização destes dispositivos prendem-se com a possível menor toxicidade do vapor gerado em comparação com o fumo do tabaco e a ausência de exposição passiva. ${ }^{32} \mathrm{O}$ facto destas hipóteses ainda se encontrarem em estudo leva a que alguns autores alertem sobretudo para o desconhecimento dos efeitos da exposição a longo prazo ao propilenoglicol, um dos componentes principais das soluções de nicotina. ${ }^{33}$

Outra preocupação frequente entre os diversos autores é que a promoção destes produtos como métodos de cessação tabágica possa afastar os fumadores da utilização de métodos comprovadamente eficazes e disponí- 
QUADRO II. Resumo das conclusões dos artigos incluídos e nível de evidência segundo a escala SORT

\begin{tabular}{l|l|l|c} 
Autor (Ano) & Tipo de estudo & Conclusão & NE \\
\hline $\begin{array}{l}\text { Grana R, et al } \\
\text { (2014) }\end{array}$ & RS com Meta-Análise & $\begin{array}{l}\text { Em relação aos estudos observacionais: utilizadores de CE apresentam uma menor } \\
\text { probabilidade de deixar de fumar tabaco } \\
\text { Em relação aos ensaios clínicos: sugerem que os CE não estão associados a um } \\
\text { maior sucesso na cessação tabágica }\end{array}$ & 2 \\
\hline $\begin{array}{l}\text { Odum LE, et } \\
\text { al (2012) }\end{array}$ & Revisão clássica & $\begin{array}{l}\text { São necessários estudos de maior qualidade para clarificar o papel dos CE na } \\
\text { cessação tabágica, até lá não podem ser recomendados }\end{array}$ & 3 \\
\hline $\begin{array}{l}\text { Brown J, et al } \\
(2014)\end{array}$ & Transversal & $\begin{array}{l}\text { Maior probabilidade de abstinência aos } 12 \text { meses nos utilizadores de CE do que } \\
\text { com a TSN }\end{array}$ & 3
\end{tabular}

Legenda: CE - Cigarros eletrónicos; NE - Nível de evidência; OR - Odds ratio; RS - Revisão sistemática; TSN - Terapêutica de substituição da nicotina.

veis, ${ }^{33}$ protelando assim a procura de tratamento adequado. Não deve também ser minimizada a proporção de indivíduos que, em alguns estudos, deixou de consumir tabaco mantendo a utilização de cigarros eletrónicos e, portanto, um consumo continuado de nicotina. ${ }^{14,27}$ Além disso, de entre os ex-fumadores que utilizam este tipo de cigarros, a maioria considera que teria uma recaída se suspendesse a sua utilização. ${ }^{13-14}$

Por outro lado, o facto de não existir restrição no acesso de menores a estes produtos, nem proibição de utilização em espaços fechados, pode promover um retrocesso de décadas no esforço de criação de uma imagem negativa associada ao ato de fumar, ${ }^{34-35}$ tornando-o novamente num hábito socialmente aceitável e atrativo para os jovens, cada vez mais expostos à publicidade agressiva destes produtos. ${ }^{33}$

Em conclusão, tendo em conta a evidência atualmente disponível, os autores consideram que o uso de cigarros eletrónicos não parece desempenhar um papel benéfico na cessação tabágica (Força de Recomendação B). Dada a rápida expansão e crescente popularidade destes produtos são necessários estudos de melhor qualidade.

\section{REFERÊNCIAS BIBLIOGRÁFICAS}

1. Dockrell M, Morison R, Bauld L, McNeill A. E-cigarettes: prevalence and attitudes in Great Britain. Nicotine Tob Res. 2013;15(10):1737-44.

2. Dawkins L, Turner J, Roberts A, Soar K. 'Vaping' profiles and preferences: an online survey of electronic cigarette users. Addiction. 2013;108(6):1115-25.

3. Goniewicz ML, Lingas EO, Hajek P. Patterns of electronic cigarette use and user beliefs about their safety and benefits: an internet survey. Drug Alcohol Rev. 2013;32(2):133-40.

4. Britton J, Bogdanovica I. Electronic cigarettes: a report commissioned by Public Health England. London: Public Health England; 2014.

5. Zhu SH, Gamst A, Lee M, Cummins S, Yin L, Zoref L. The use and perception of electronic cigarettes and snus among the U.S. population. PLoS One. 2013;8(10):e79332.

6. McArdle M. E-cigarettes: a $\$ 1.5$ billion industry braces for FDA regulation. Bloomberg Business; 2014 Feb 6 [cited 2014 Jun 25]. Available from: http://www.businessweek.com/articles/2014-02-06/e-cigarettes-fda-regulation-looms-for1-dot-5-billion-industry

7. King BA, Alam S, Promoff G, Arrazola R, Dube SR. Awareness and ever-use of electronic cigarettes among U.S. adults, 2010-2011. Nicotine Tob Res. 2013;15(9):1623-7.

8. TNS Opinion \& Social. Attitudes of Europeans towards tobacco: special Eurobarometer 385. Brussels: European Commission; 2012.

9. Choi K, Forster J. Characteristics associated with awareness, perceptions, and use of electronic nicotine delivery systems among young US Midwestern adults. Am J Public Health. 2013;103(3):556-61.

10. Ayers JW, Ribisl KM, Brownstein JS. Tracking the rise in popularity of electronic nicotine delivery systems (electronic cigarettes) using search query surveillance. Am J Prev Med. 2011;40(4):448-53.

11. Grana RA, Ling PM. "Smoking revolution": a content analysis of electronic cigarette retail websites. Am J Prev Med. 2014;46(4):395-403.

12. Wagener TL, Siegel M, Borrelli B. Electronic cigarettes: achieving a balanced perspective. Addiction. 2012;107(9):1545-8.

13. Etter JF, Bullen C. Electronic cigarette: users profile, utilization, satisfaction and perceived efficacy. Addiction. 2011;106(11):2017-28.

14. Foulds J, Veldheer S, Berg A. Electronic cigarettes (e-cigs): views of aficionados and clinical/public health perspectives. Int J Clin Pract. 2011;65(10):1037-42.

15. Pearson JL, Richardson A, Niaura RS, Vallone DM, Abrams DB. e-Cigarette awareness, use, and harm perceptions in US adults. Am J Public Health. 2012;102(9):1758-66.

16. Diretiva $n^{\circ}$ 2014/40/EU, do Parlamento Europeu e do Conselho, de 3 de abril de 2014, relativa à aproximação das disposições legislativas, regulamentares e administrativas dos Estados-Membros no que respeita ao fabrico, apresentação e venda de produtos do tabaco e produtos afins e que revoga a Diretiva 2001/37/CE. JOUE. 2014 Abr 29:L(127):1-38.

17. Ebell MH, Siwek J,Weiss BD, Susman J, Ewigman B, Bowman M. Strength of recommendation taxonomy (SORT): a patient-centered approach to grading evidence in the medical literature. Am Fam Physician. 2004;69(3):548-56.

18. Jadad AR, Moore RA, Carroll D, Jenkinson C, Reynolds DJ, Gavaghan DJ, et al.Assessing the quality of reports of randomized clinical trials: is blinding necessary? Control Clin Trials. 1996;17(1):1-12. 
19. Wells G, Shea B, O'Connell D, Robertson J, Peterson J, Welch V, et al. Newcastle-Ottawa Scale (NOS) for assessing the quality of nonrandomized studies in meta-analysis [Internet]. Ottawa: Ottawa Health Research Institute; 2009. Available from: http://www.evidencebasedpublichealth.de/download/Newcastle_Ottowa_Scale_Pope_Bruce.pdf

20. Grana R, Benowitz N, Glantz SA. E-cigarettes: a scientific review. Circulation. 2014;129(19):1972-86.

21. Odum LE, O'Dell KA, Schepers JS. Electronic cigarettes: do they have a role in smoking cessation? J Pharm Pract. 2012;25(6):611-4.

22. Brown J, Beard E, Kotz D, Michie S, West R. Real-world effectiveness of e-cigarettes when used to aid smoking cessation: a cross-sectional population study Addiction. 2014;109(9):1531-40.

23. Yao T, Jiang N, Grana R, Ling PM, Glantz SA. A content analysis of electronic cigarette manufacturer websites in China. Tob Control. 2014 Oct 21. doi: 10.1136/tobaccocontrol-2014-051840

24. Grana RA, Ling PM, Benowitz N, Glantz M. Electronic cigarettes: cardiology patient page. Circulation. 2014;129(19):e490-2.

25. Lazar P, Granatier J, Klimes J, Hobza P, Otyepka M. The nature of bonding and electronic properties of graphene and benzene with iridium adatoms. Phys Chem Chem Phys. 2014;16(38):20818-27.

26. Grana RA, Popova L, Ling PM. A longitudinal analysis of electronic cigarette use and smoking cessation. JAMA Intern Med. 2014;174(5):812-3.

27. Bullen C, Howe C, Laugesen M, McRobbie H, Parag V, Williman J, et al. Electronic cigarettes for smoking cessation: a randomised controlled trial. Lancet. 2013;382(9905):1629-37.

28. University of Michigan Health System.Tobacco treatment.AnnArbor, MI:UCHS; 2012 [cited 2014 Oct 11]. Available from: http://www.guideline.gov/content.aspx?id=36845

29. Yamin CK, Bitton A, Bates DW. E-cigarettes: a rapidly growing Internet phenomenon. Ann Intern Med. 2010;153(9):607-9.
30. Infarmed. Cigarros electrónicos: circular informativa n 156/CD, de 12/08/2011. Lisboa: Infarmed; 2011.

31. Garcia R. Empresários dizem que estão atentos às preocupações quanto ao cigarro electrónico. Público. 2014 Aug 27. Available from: http://www.publico.pt/ecosfera/noticia/empresarios-dizem-que-estao-atentos-as-preocupacoes-quanto-ao-cigarro-electronico-1667798

32. Goniewicz ML, Knysak J, Gawron M, Kosmider L, SobczakA, Kurek J, et al. Levels of selected carcinogens and toxicants in vapour from electronic cigarettes. Tob Control. 2014;23(2):133-9.

33. Cobb NK, Abrams DB. E-cigarette or drug-delivery device? Regulating novel nicotine products. N Eng J Med. 2011;365(3):193-5.

34. Katz MH. If only electronic cigarettes were effective smoking cessation devices. JAMA Intern Med. 2014;174(5):813-4.

35. Chapman S. Should electronic cigarettes be as freely available as tobacco cigarettes? No. BMJ. 2013;346:f3840.

\section{CONFLITO DE INTERESSES}

Os autores declaram não ter conflitos de interesses.

\section{ENDEREÇO PARA CORRESPONDÊNCIA}

Pedro Daniel Miranda Couto

USF Valongo, Rua da Misericórdia, s/n, 4440-563 Valongo

Email: couto.pedro@gmail.com

Recebido em 02-12-2014

Aceite para publicação em 01-06-2015

\section{ABSTRACT}

\section{THE ROLE OF ELECTRONIC CIGARETTES IN SMOKING CESSATION: AN EVIDENCE-BASED REVIEW}

Objectives: To determine if electronic cigarettes are effective in smoking cessation.

Data sources: MEDLINE databases; National Clearinghouse; Canadian Medical Association Practice Guidelines InfoBase; Guidelines Finder of the National Electronic Library for Health in the British NHS; Database of Abstracts of Reviews of Effectiveness - Centre for Reviews and Dissemination; Bandolier; The Cochrane Library; Index of Portuguese Medical Journals.

Review methods: Articles published between 01/07/2004 and 30/06/2014 using the MeSH term 'smoking cessation', the English terms 'electronic cigarette(s)', 'e-cigarette(s)', 'e-cig(s)' and the Portuguese terms 'cessação tabágica' and 'cigarro(s) ele(c)trónico(s)'. Studies that evaluated the smoking cessation in smokers exposed and not exposed to electronic cigarettes were included. 'Strength-of-Recommendation Taxonomy','Jadad' and 'Newcastle-Ottawa' scales were used to assess the quality of the studies and the strength of recommendations.

Results: The search produced a list of 2,076 articles. Ten articles were excluded as duplicates, 2,005 were excluded after reading the title because they were not directly related to the topic, 47 were excluded after reading the summary, and 11 after reading the full text because they did not meet the inclusion criteria. Three articles were included in this review. They were a systematic review, which included nine original studies [Level of Evidence (LE) 2], a classic review (LE 3), whose conclusions were unfavorable to the use of electronic cigarettes, and one cross-sectional study, which showed an increased use of electronic cigarettes compared to other therapies among those who stopped smoking (LE 3). Conclusions: Most studies found no association between use of electronic cigarettes and smoking cessation. The authors concluded that its use does not seem to play a beneficial role in smoking cessation (Strength of Recommendation B). Given the rapid expansion and growing popularity of these products, better quality studies are needed.

Keywords: Electronic Cigarette; Smoking Cessation. 